\title{
Microduplication of the ARID1A gene causes intellectual disability with recognizable syndromic features
}

\author{
Marie Bidart, PharmD, PhD ${ }^{1,2}$, Michèle El Atifi, $\mathrm{PhD}^{1,2}$, Sarra Miladi, MS ${ }^{1,2}$, \\ John Rendu, PharmD, PhD 2,3, Véronique Satre, MD, PhD 2,4,5, Pierre F. Ray, PhD 2,3,5, \\ Caroline Bosson, PharmD³, Françoise Devillard, $\mathrm{MD}^{4}$, Daphné Lehalle, MD ${ }^{6}$, Valérie Malan, MD, $\mathrm{PhD}^{7}$, \\ Jeanne Amiel, MD, $\mathrm{PhD}^{6}$, Maria Antonietta Mencarelli, MD, $\mathrm{PhD}^{8}$, Margherita Baldassarri, $\mathrm{MD}^{8,9}$, \\ Alessandra Renieri, MD, PhD ${ }^{8,9}$, Jill Clayton-Smith, MD, PhD ${ }^{10}$, Gaëlle Vieville, MS ${ }^{4}$, \\ Julien Thevenon, MD, PhD ${ }^{11}$, Florence Amblard, MD ${ }^{4}$, François Berger, $\mathrm{MD}, \mathrm{PhD}^{1,2}$, \\ Pierre-Simon Jouk, MD, $\mathrm{PhD}^{2,4}$ and Charles Coutton, PharmD, PhD $2,4,5$
}

Purpose: To determine whether duplication of the ARID1A gene is responsible for a new recognizable syndrome.

Methods: We describe four patients with a 1p36.11 microduplication involving ARID1A as identified by array-comparative genomic hybridization. We performed comparative transcriptomic analysis of patient-derived fibroblasts using RNA sequencing and evaluated the impact of ARID1A duplication on the cell cycle using fluorescenceactivated cell sorting. Functional relationships between differentially expressed genes were investigated with ingenuity pathway analysis (IPA).

Results: Combining the genomic data, we defined a small (122 kb), minimally critical region that overlaps the full ARID1A gene. The four patients shared a strikingly similar phenotype that included intellectual disability and microcephaly. Transcriptomic analysis revealed the deregulated expression of several genes previously linked to microcephaly and developmental disorders as well as the involvement of signaling pathways relevant to microcephaly, among which the polo-like kinase (PLK) pathway was especially notable. Cell-cycle analysis of patient-derived fibroblasts showed a significant increase in the proportion of cells in G1 phase at the expense of G2-M cells.

Conclusion: Our study reports a new microduplication syndrome involving the ARID1A gene. This work is the first step in clarifying the pathophysiological mechanism that links changes in the gene dosage of ARID1A with intellectual disability and microcephaly.

Genet Med advance online publication 1 December 2016

Key Words: 1p36.11 microduplication; ARID1A; intellectual disability; microcephaly; RNA sequencing

\section{INTRODUCTION}

ARID1A (AT-rich interactive domain-containing protein 1A), also known as BAF250A, is one of the main subunits of the switch/sucrose nonfermentable (SWI/SNF)-like chromatinremodeling complex (BAF complex), a multiprotein complex essential for chromatin remodeling. This complex plays a key role in the epigenetic regulation of genome expression and has been implicated in several cellular functions, including proliferation, self-renewal of stem cells, DNA methylation, and damage repair. ${ }^{1}$ In particular, the ARID1A-containing chromatin-remodeling complex regulates processes related to tumor suppression, ${ }^{2}$ and somatic mutations that inactivate the ARIDIA gene have been identified in a wide spectrum of human neoplasms. ${ }^{3}$ Moreover, ARID1A shows one of the highest mutation rates across different types of human cancer. ${ }^{4}$

Recent discoveries of ARID1A germ-line mutations associated with Coffin-Siris syndrome (CSS; OMIM 135900) demonstrated that ARID1A also plays a critical role in human neuronal development. ${ }^{5}$ CSS is a rare autosomal-dominant syndrome characterized by intellectual disability, growth deficiency, microcephaly, coarse facial features, and hypoplastic nails, especially on the fifth finger and/or toe. Other findings commonly include feeding difficulties, ophthalmologic abnormalities, brain malformations, and hearing loss. ${ }^{6,7}$ Large-scale

\footnotetext{
${ }^{1}$ UF Clinatec, Pôle Recherche, INSERM UMR 1205, CHU de Grenoble, Grenoble, France; ${ }^{2}$ Université Grenoble-Alpes, Grenoble, France; ${ }^{3}$ Département de Biochimie Toxicologie et Pharmacologie, Département de Biochimie Génétique et Moléculaire, Centre Hospitalier Universitaire de Grenoble, Grenoble, France; ${ }^{4}$ Département de Génétique et Procréation, Hôpital Couple-Enfant, CHU de Grenoble, Grenoble, France; ${ }^{5}$ Equipe "Genetics Epigenetics and Therapies of Infertility," Institut Albert Bonniot, INSERM U823, La Tronche, France; ${ }^{6}$ Service de Génétique, INSERM U781, Hôpital Necker-Enfants Malades, Institut Imagine, University Sorbonne-Paris-Cité, Paris, France; ${ }^{7}$ Service de Cytogénétique et UMR_S1163, IHU Imagine, Hôpital Necker-Enfants Malades, Paris, France; ${ }^{8}$ Genetica Medica, Azienda Ospedaliera Universitaria Senese, Siena, Italy; ${ }^{9}$ Medical Genetics, University of Siena, Siena, Italy; ${ }^{10}$ Manchester Centre for Genomic Medicine, Central Manchester University Hospitals, Manchester Academic Health Sciences Centre, Manchester, UK; ${ }^{11}$ Centre de Génétique et Centre de Référence “Anomalies du Développement et Syndromes Malformatifs," Hôpital d’Enfants, CHU Dijon, Dijon, France. Correspondence: Charles Coutton (ccoutton@chu-grenoble.fr)
} 


\section{ORIGINAL RESEARCH ARTICLE}

studies reported that 41 to $100 \%$ of CSS subjects carry a germline mutation in one of the BAF complex subunit genes., ${ }^{5,8-12}$ Overall, mutations in the ARID1A gene are found in only $7.5 \%$ of cases, a lower frequency than that observed for the other genes of the BAF complex. ${ }^{13}$ All CSS-causing mutations previously identified in ARIDIA are heterozygous and have a lossof-function effect, indicating that ARID1A is a dosage-sensitive gene. ${ }^{5}$ Therefore, it raises the possibility that the gain of a copy of ARIDIA could also be pathogenic. We previously identified a microduplication encompassing the ARIDIA gene in a girl presenting with intellectual disability and microcephaly. ${ }^{14}$ In the present report, we compare the genomic data and clinical features of four patients carrying a small 1p36.11 microduplication encompassing the complete ARID1A gene. Subsequent molecular studies then allowed us to explore the functional pathways and biological functions disturbed by ARID1A duplication.

\section{MATERIALS AND METHODS}

\section{Patients}

Following the diagnosis of our first patient, ${ }^{14}$ three additional patients were identified as harboring small 1p36.11 duplications that fully included ARIDIA; they were recruited through the DECIPHER database. To limit the number of genes encompassed to be able to establish a precise genotype-phenotype correlation, only duplications smaller than $1 \mathrm{Mb}$ were selected. Clinical information was gathered through a detailed questionnaire addressed to the referring clinician. An overview of these four patients (PT1, PT2, PT3, and PT4) and their clinical characteristics are presented in Table 1. A more detailed clinical report for each patient can be found in the Supplementary Clinical Information online. Photographs of patients (PT1-PT4) are shown in Figure 1a. Two patients were from France (PT1 and PT2), one from Italy (PT3), and one from the United Kingdom (PT4). Written informed consent for publication of their clinical details and/or clinical images was obtained from all patients and institutional approval was given by the local medical ethical committee (Grenoble Institutional Review Board, 6705). We also included three healthy volunteers (C1-C3) without ARID1A duplication (data not shown) as control samples. Control subjects were recruited in France with self-declared European ancestry. Two were females, aged 18 and 25 , respectively, and one was a 22 -years-old male. Written informed consent was obtained from all controls.

\section{Chromosomal microarray analysis}

DNA was extracted from peripheral blood lymphocytes of the patients following standard protocols. All four 1p36.11 microduplications were detected using a 60,000-oligonucleotide microarray (Human Genome CGH Microarray Kit 60K; Agilent Technologies, Santa Clara, CA) according to the manufacturer's instructions. The Database of Genomic Variants (http://projects.tcag.ca/ variation/) was used to compare our findings to those of previously reported studies. Genomic positions are based on the UCSC GRCh37/hg19 assembly. Verifications of gain of 1 p36.11 material in the proband and parental testing were subsequently performed as described in the Supplementary Methods online.

\section{Skin biopsies}

Skin biopsies were performed for PT1, PT2, and PT3 and the three control individuals (C1-C3). Samples were obtained from a cutaneous biopsy of the forearm skin using a scalpel and biopsy forceps (size $\approx 0.5 \mathrm{~mm}^{3}$ ). Only fibroblast cultures from PT1 and PT2 were included in our study because fibroblasts from PT3 did not multiply in vitro.

\section{Cell culturing}

Biopsy tissue was rinsed several times with an antibiotic-antimycotic solution. Tissue was then placed in $1 \mathrm{ml}$ of collagenase solution and incubated for $24 \mathrm{~h}$ at $37{ }^{\circ} \mathrm{C}$ in a $5 \% \mathrm{CO}_{2}$ atmosphere. The resulting confluent culture was designated passage 1. Cells were then split to generate three $25-\mathrm{cm}^{2}$ flasks. Cultures at $70 \%$ confluence were harvested by centrifugation $(340 \mathrm{~g})$, and cell pellets were stored at $-80^{\circ} \mathrm{C}$ in RNAlater (Ambion, Cambridge, MA).

\section{Next-generation sequencing and data analysis}

RNA paired-end sequencing (RNA-seq) was performed for two patients (PT1 and PT2) and three controls with RNA from skin fibroblasts. The functional relationships between genes differentially expressed between patients and controls were subsequently investigated using Ingenuity Pathway Analysis (IPA) software (Qiagen, Hilden, Germany, https://analysis.ingenuity.com). Further details are provided in the Supplementary Methods online.

\section{Quantitative reverse-transcription polymerase chain reaction}

Quantitative reverse-transcription polymerase chain reaction (qRT-PCR) experiments were first performed using two candidate genes: ARIDIA and PIGV. Then, the expression of the various deregulated genes was verified by qRT-PCR to confirm the results observed with RNA sequencing. Validation was applied to a subset of 24 genes that were differentially expressed by at least fourfold in patient-derived fibroblasts using RNA-seq. Because the mitotic role of polo-like kinase pathway was among the most statistically significant pathways deregulated in our patients, we decided to test the expression of PLK1, another key gene of the PLK pathway, which was not initially detected with RNA-seq in PT1, probably owing to technical failure. qRT-PCR was performed as described in the Supplementary Methods online. The primer sequences and qRT-PCR protocol are presented in Supplementary Table S1 online.

\section{Western blot analysis}

We performed western blot analyses of ARID1A and PLK4 in patient and control fibroblasts. Total protein was extracted from cells in RIPA lysis buffer (R-078; Sigma-Aldrich, St. Louis, MO). Western blotting was performed on NuPAGE Novex 4-12\% Bis-Tris Mini Gels (Thermo Fisher Scientific, Waltham, $\mathrm{MA})$. The membranes were incubated with primary antibody 
Table 1 Comparison of the clinical features and molecular data of the four patients presented in this study

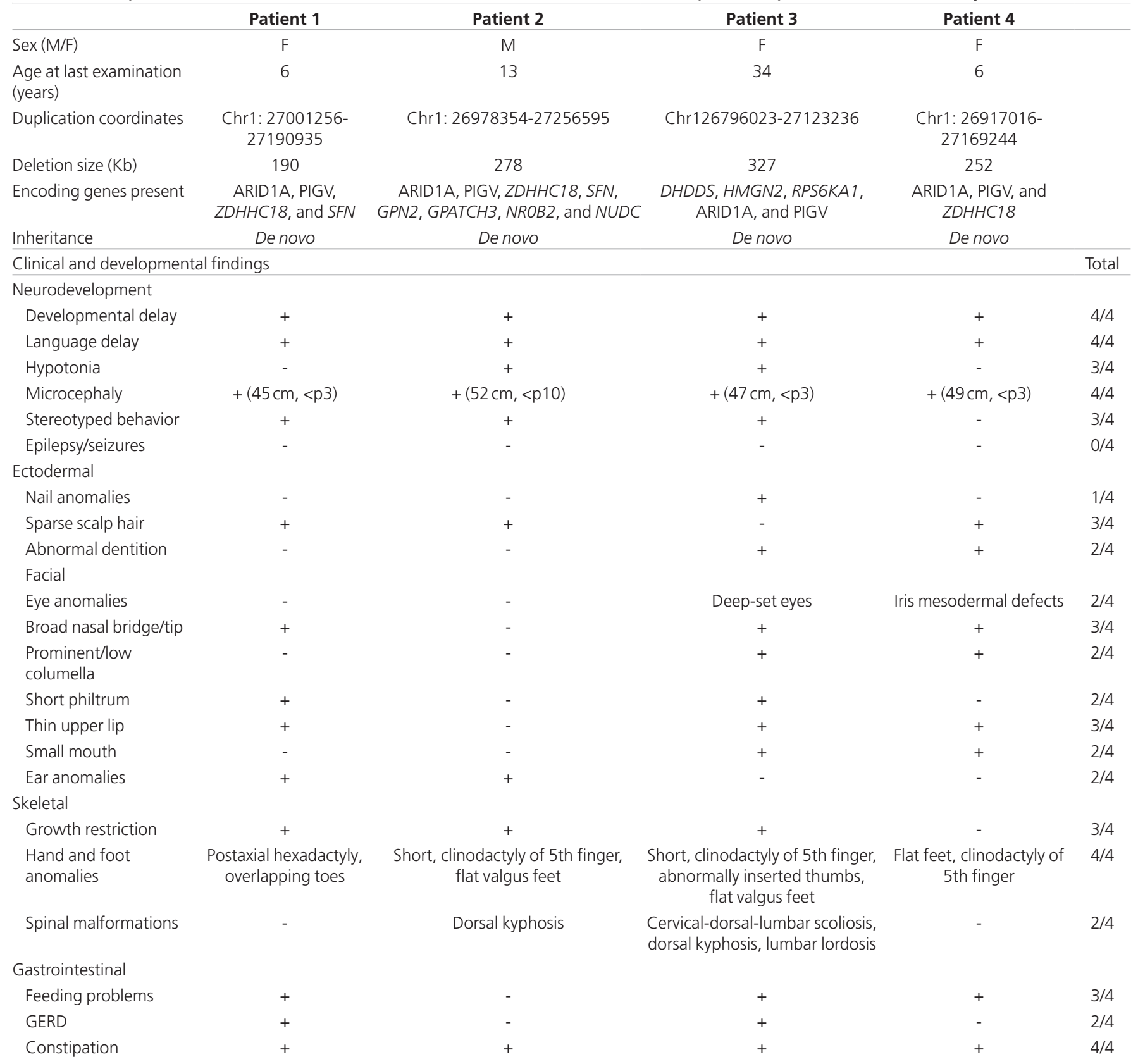

Cold hand and feet Recurrent airway infections, PA-VSD, Recurrent airway infections, Recurrent airways syndrome short neck, vasomotor problems edema of lower limbs, centripetal infections, hoarse voice obesity

Coordinates of all variations are based on the UCSC GRCh37/hg19 assembly.

+, Feature present; -, feature absent; GERD, gastroesophageal reflux disease; p, percentile; PA-VSD, pulmonary atresia with ventricular septal defect.

(anti-ARID1A: ab71394; Abcam, Cambridge, UK; anti-PLK4: ab71394; Abcam).

siRNA-mediated ARID1A silencing in fibroblast cells

At 1 day after plating, fibroblasts derived from PT1 and PT2 were transfected with $10 \mathrm{nmol} / \mathrm{l}$ of ARID1A-targeted short interfering RNA (siRNA), using Lipofectamine RNAiMAX Reagent (Life Technologies, Carlsbad, CA) according to the manufacturer's recommendations. ARID1A-targeted siRNA was purchased from Ambion (AM16708, ID 147403; Life Technologies). A Blast search on the NCBI database ensured specific targeting to ARID1A mRNA. We used Silencer Select 
a
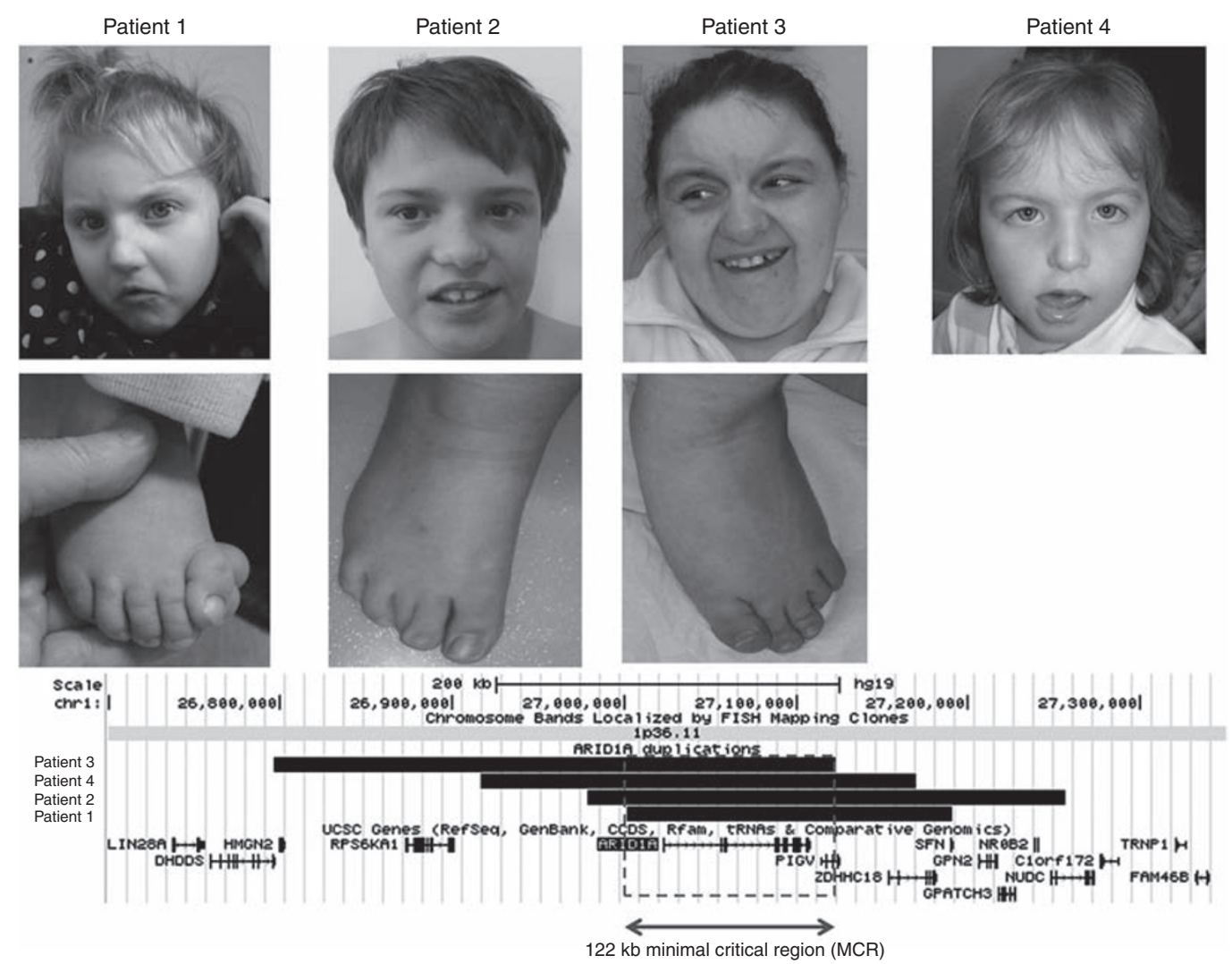

Figure 1 Photographs and genomic rearrangements in patients with ARID1A duplications (PT1-PT4). (a) Patient 1 (PT1), 6 years old; patient 2 (PT2), 13 years old; patient 3 (PT3), 34 years old; and patient 4 (PT4), 10 years old. All patients presented with intellectual disability and microcephaly and have a characteristic nasal tip with notched alae nasi and a low columella. The feet are very distinctive, with brachydactyly and shortening and medial deviation of the great toes. (b) Alignment of the genomic rearrangements reported in the four patients (PT1-PT4) with 1p36.11 duplications. A small (122 kb), minimally critical region (MCR; gray dash line box) (chr1:27,001,256-27,123,236) common to all four index cases was defined. Among the duplicated genes in the MCR, only the ARID1A gene was completely duplicated. A second gene, PIGV, was partially included.

Negative Control No. 1 siRNA (AM17140; Life Technologies) as scrambled nontargeting siRNA (negative control). RNA extractions were performed $48 \mathrm{~h}$ after siRNA transfection. Finally, we performed subsequent qRT-PCR for a subset of 10 PLK pathway genes that were differentially expressed by at least fourfold in patient-derived fibroblasts using RNA-seq.

\section{Cell-cycle analysis}

We performed cell-cycle analysis for patients PT1 and PT2 and for the control pool (C1-C3). A total of $5 \times 10^{6}$ cells were harvested, rinsed with cold phosphate-buffered saline (PBS), and fixed with absolute ice-cold ethanol at $-20^{\circ} \mathrm{C}$. Fixed cells were rinsed with cold PBS, followed by incubation with PBS containing $500 \mu \mathrm{g} / \mathrm{ml}$ propidium iodide and $1 \mathrm{mg} / \mathrm{ml} \mathrm{RNase} \mathrm{A}$ for $1 \mathrm{~h}$ at $37^{\circ} \mathrm{C}$. The DNA content of labeled cells was acquired using fluorescence-activated cell sorting with a BD FACSCalibur flow cytometer (BD Biosciences, Le Pont de Claix, France). Each experiment was performed in triplicate.

\section{CMA analysis}

\section{RESULTS}

Array-comparative genomic hybridization analyses of the four patients showed a genomic duplication in chromosome band 1p36.11. For all these patients, no other chromosomal anomalies larger than three probes were observed, excluding wellknown benign copy-number variations. All duplications were confirmed and occurred de novo. These aberrations ranged in size from 190 to $327 \mathrm{~kb}$ and contained three to eight genes (Figure 1b). All aberrations led to a complete duplication of ARID1A. Together, these duplications defined a minimal critical region (MCR) of $122 \mathrm{~kb}$ (chr1:27,001,256-27,123,236) that fully included the ARID1A gene and partially included the PIGV gene (Figure 1b).

\section{Overview of differentially expressed genes induced by ARID1A microduplication}

The overview of differentially expressed genes is based on the analysis of two patients, PT1 and PT2, and three controls. We identified 2,890 differentially expressed genes between patients and controls, including 1,325 downregulated genes (fold change $<0.5$ ) and 1,535 upregulated genes (fold change $>2$ ). According to our IPA criteria, we identified five top canonical pathways in which the uploaded gene set participated more than could be expected by chance $(P>3)$ : cell-cycle control of chromosomal replication $(P=7.37)$, hereditary breast cancer signaling 
$(P=5.48)$, role of BRCA1 in DNA damage response $(P=5.29)$, mitotic role of polo-like kinase $(P=4.96)$, and ataxia telangiectasia mutated protein signaling $(P=3.54)$. Of these top five pathways, only the mitotic role of the polo-like kinase pathway showed a significant absolute $z$-score $>2$ (2.673) (Figure 2a).
Next, we examined diseases and biological functions overrepresented in our data. We classified items according to the best $P$-value with a significant absolute $z$-score $(\geq 2)$ (Supplementary Table S2 online). The top-ranked biological functions included the general categories of cell death and

a

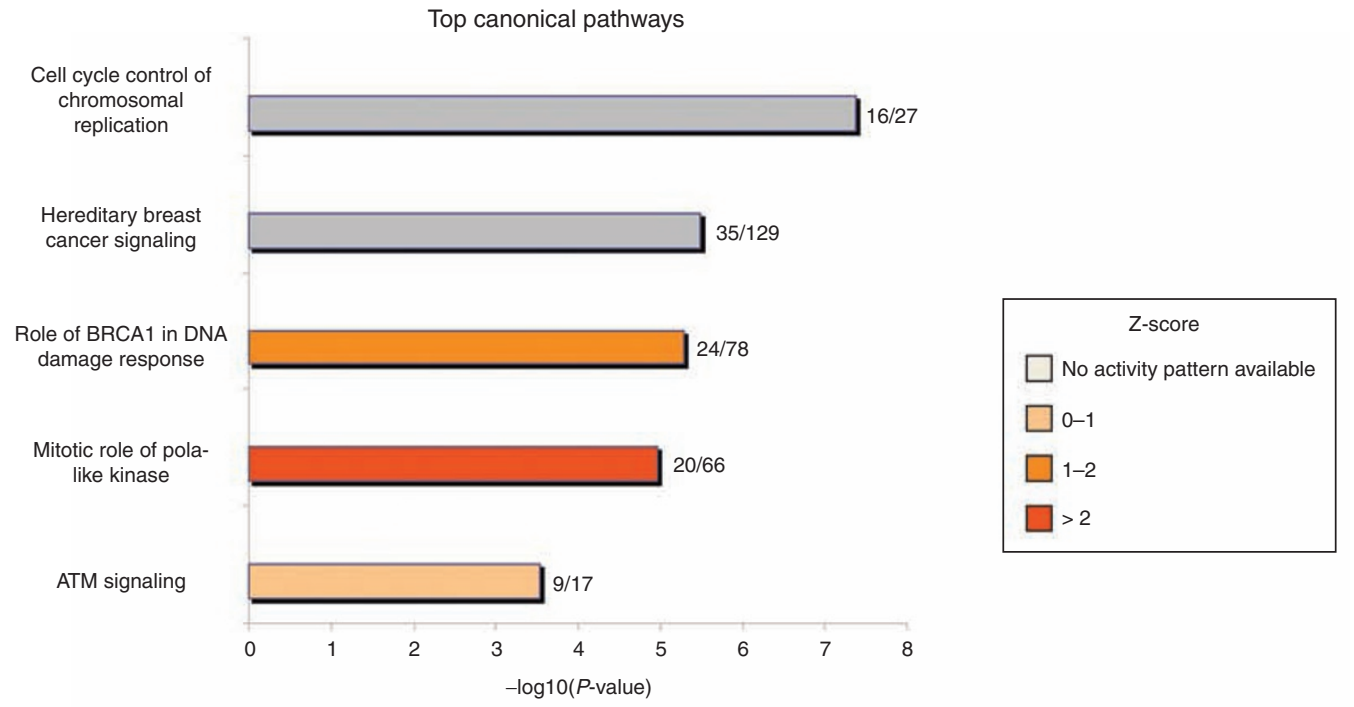

b

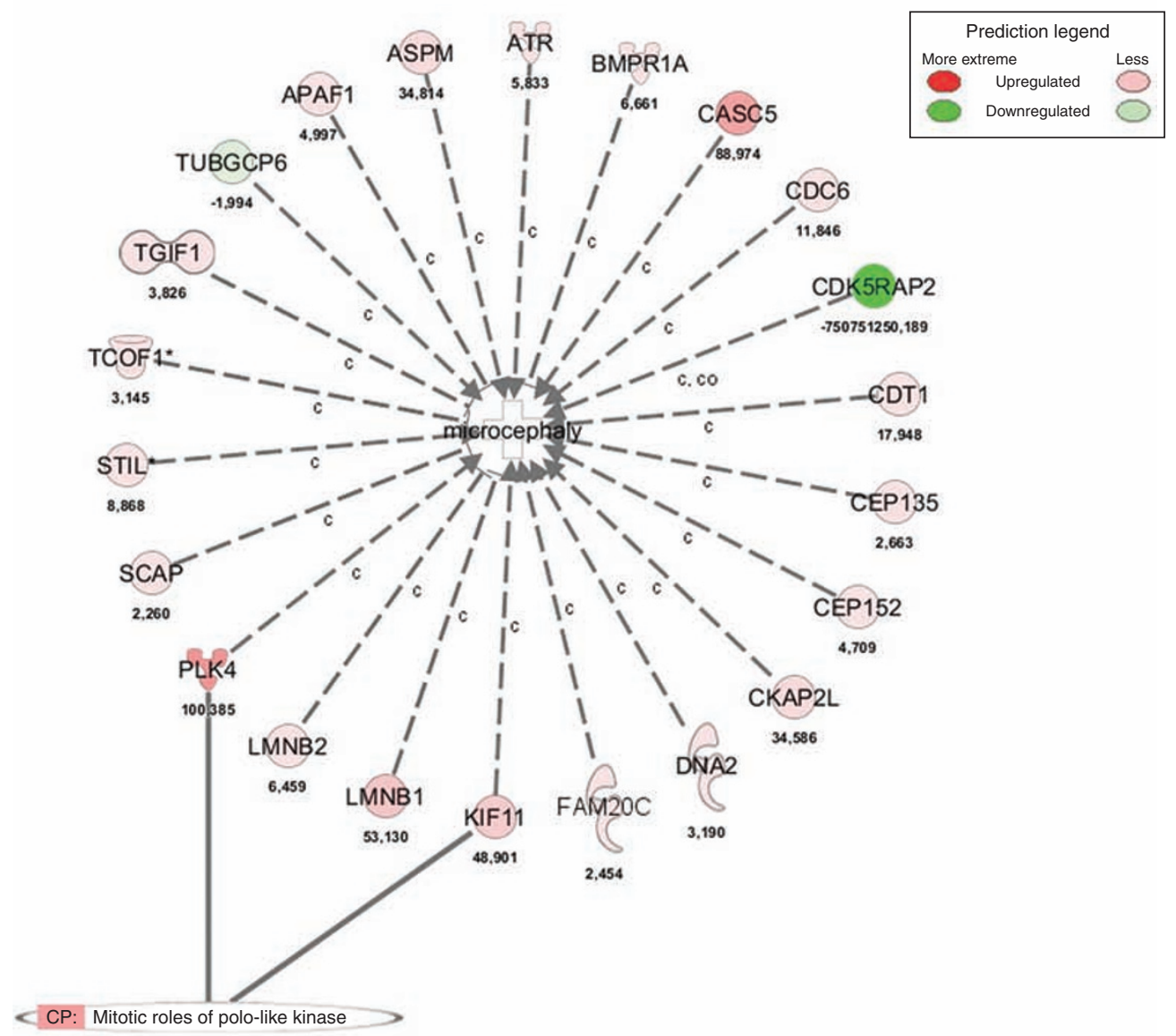

Figure 2 RNA sequencing results. (a) The five canonical pathways identified by ingenuity pathway analysis (IPA) as significantly ( $P>3$ ) associated with the set of genes deregulated between patients and controls. The absolute $z$-score as well as the number of genes differentially expressed among the total genes assigned to the canonical pathway by IPA are indicated. (b) Variations in the expression of genes implicated in microcephaly observed in patient-derived fibroblasts. Red genes are upregulated genes and green genes are downregulated genes. 
survival, cell cycle, and cell growth and proliferation, as well the more specific category of DNA replication, recombination, and repair. Detailed biological functions are presented in Supplementary Table S2 online. Regarding diseases, the topranked categories were cancer, which showed high levels of enrichment (up to $P=2.51 \mathrm{e}^{-10}$ ), and developmental disorders. Of these developmental disorders, IPA predicted a significant enrichment of genes related to congenital malformations of the skeleton $\left(P=4.13 \mathrm{e}^{-6}, z\right.$-score: 2.146$)$, craniofacial abnormalities $\left(P=5.49 \mathrm{e}^{-6}, z\right.$-score: 2.176$)$, growth failure $\left(P=6.16 \mathrm{e}^{-5}\right.$, $z$-score: 4.002$)$, microcephaly $\left(P=8.54 \mathrm{e}^{-5}, z\right.$-score: 2.630$)$, and congenital malformations of the brain $\left(P=2.76 \mathrm{e}^{-4}, z\right.$-score: 2.534) (Supplementary Table S2 online). We subsequently investigated the microcephaly gene network and observed that the polo-like kinase 4 (PLK4) gene was the most strongly deregulated gene (Figure $\mathbf{2 b}$ ). We also noted that the PLK4 gene harbors the strongest overexpression in the mitotic role of the polo-like kinase pathway (Supplementary Table S3 online).

\section{Validation of the differential expression of selected genes by qRT-PCR}

qRT-PCR was performed for the two genes (ARIDIA and PIGV) in the 122-kb MCR. Only the expression of ARID1A increased approximately 1.5 -fold compared to controls (Supplementary Table S4 online), whereas PIGV expression did not seem to be altered. qRT-PCR validation was also applied to a subset of 25 genes, including PLK1. Eleven of the 25 selected genes belonged to the mitotic role of polo-like kinase pathway, and each showed significantly increased expression in patient-derived fibroblasts compared to control-derived fibroblasts (Figure 3a and Supplementary Table S4 online). We confirmed that PLK4 expression appeared strongly upregulated in the two patients a

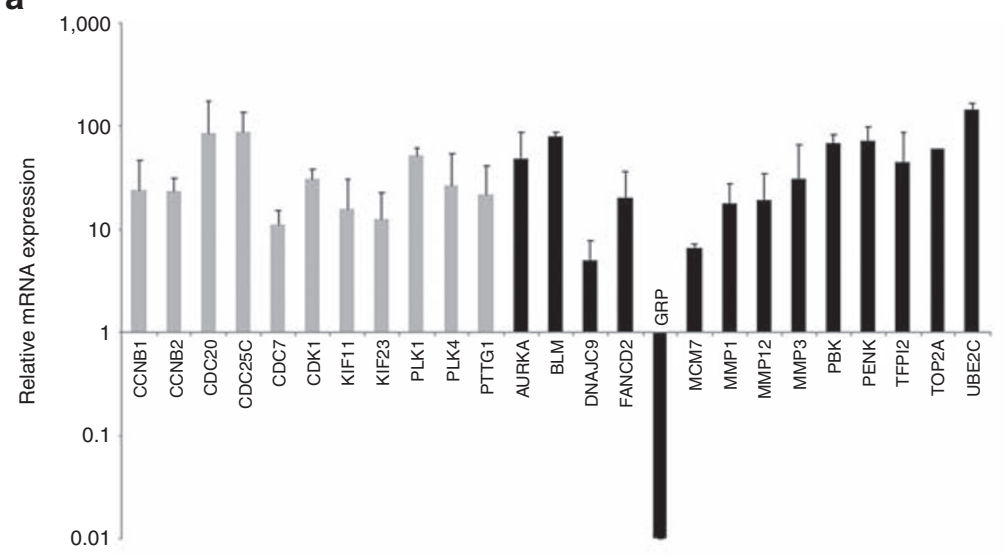

b

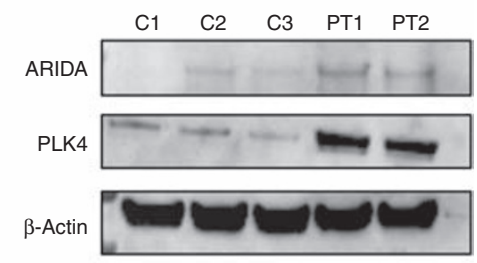

C

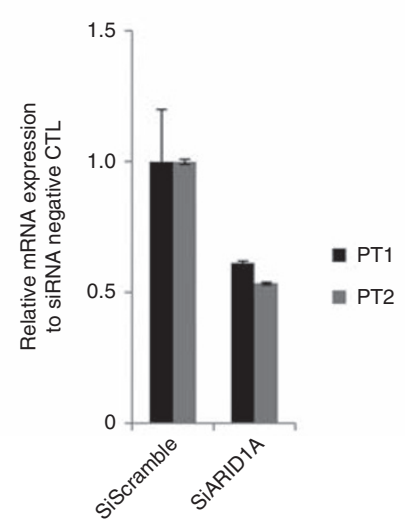

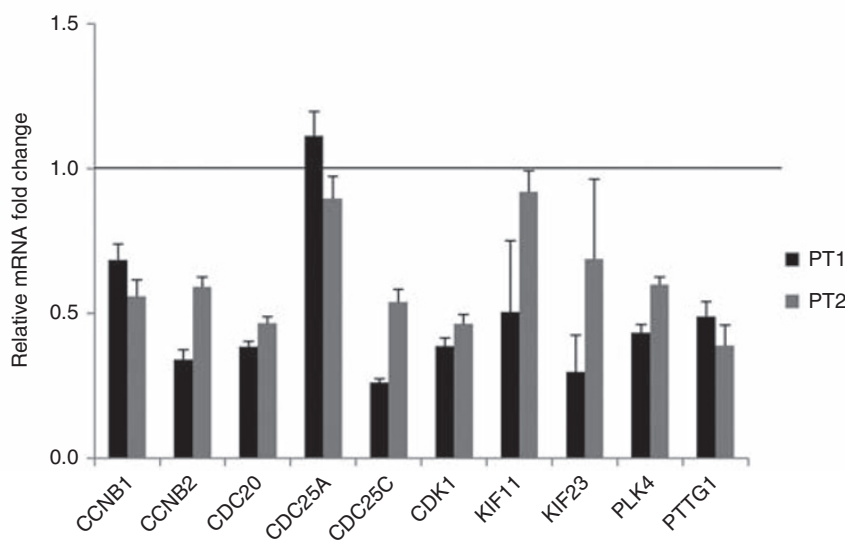

Figure 3 Validation of deregulated genes derived from RNA-seq analysis. (a) Validation of gene expression values derived from RNA-seq by qRT-PCR. qRT-PCR was applied to a subset of 25 genes. Eleven of the 25 selected genes belonged to the mitotic role of polo-like kinase pathway (gray bars). ARN samples from patient 1 (PT1) and patient 2 (PT2) were tested independently and compared to the control pool (C1-C3). Data are presented as the mean of the experiments \pm standard deviation for the two patients. QRT-PCR data were normalized using two reference genes, ribosomal protein L27 (RPL27) and glyceraldehyde-3-phosphate dehydrogenase (GAPDH), with the $-\triangle \triangle C$ t method. (b) Western blot analyses of ARID1A and PLK4 in patient and control fibroblasts. Endogenous expression of ARID1A and PLK4 were examined in PT1 and PT2 and control fibroblasts (C1-C3) by western blot. Western blot analyses showed that ARID1A expression is slightly increased, whereas PLK4 is markedly increased in PT1 and PT2 cells. Beta-actin served as a loading control. (c) siRNA experiments targeting ARID1A in patient-derived fibroblasts. The left panel shows the relative gene expression of ARID1A after siRNA transfection in PT1 and PT2 fibroblasts. The right panel shows the relative gene expression of the subset of PLK pathway genes after siRNA targeted against the ARID1A. The gray line indicates the normalized level of negative siRNA control. Expression of GAPDH and RPL27 was used as a normalization control. Data were pooled from two individual experiments containing three replicates per condition. Error bars show SEM. 
with ARID1A duplication (mean FC: 31) (Figure 3a and Supplementary Table S4 online). We also tested the expression of PLK1, which was initially not detected with RNA-seq in PT1. qRT-PCR showed a strong increase in PLK1 expression in PT1 (FC: 44) and in PT2 (FC: 59). qRT-PCR experiments were performed for 14 other genes assigned to different pathways and confirmed the significant gene expression alterations observed with RNA sequencing. We were able to identify a disparity in the fold-change values calculated using qRT-PCR and RNA-seq. This could be due to the normalization process of data analysis. RNA-seq allows absolute quantitation of mRNA levels, whereas our quantitative PCR experiments used relative quantification. qRT-PCR is able to detect the main mRNA and some specific splice variants of our selected genes, but additional variants should exist; they can be detected and quantified only by RNA-seq. Despite such differences in the fold-change values, our data showed that the qRT-PCR results were consistent with the RNA-seq results for all 25 selected genes, confirming that comparative RNA-seq analysis is a reliable quantitative approach.

\section{Western blot analyses}

Western blot analyses showed that ARID1A and PLK4 protein expressions were increased cells in PT1 and PT2 (Figure 3b). ARID1A protein expression was slightly but significantly increased in patient cells, which is consistent with the 1.5-fold overexpression detected by quantitative PCR. PLK4 expression was markedly increased in patient cells, confirming the strong PLK4 overexpression evidenced by the quantitative PCR and RNA-seq data.

\section{In vitro inhibition of ARID1A expression by siRNA}

ARID1A-targeted siRNA experiments were performed in cultured cells from PT1 and PT2 and led to a twofold decrease of ARID1A expression (Figure 3c). The decrease in the expression of $A R I D 1 A$ caused significant downregulation of the expression of almost all PLK pathway genes tested by
qRT-PCR (Figure 3c). This result demonstrated that variations of ARID1A gene expression could modulate the expression of several PLK pathway genes and strongly supports the idea that genomic ARID1A duplication could be directly responsible for the dysregulated mitotic role of the polo-like kinase pathway observed with RNA-seq in patient fibroblasts.

\section{Cell-cycle perturbation}

We examined the cell-cycle distribution by flow cytometry on control and patient fibroblasts. The distribution of the cell-cycle phases based on analysis using BD Accuri C6 Flow Cytometry software is shown in Figure 4. PT1 and PT2 fibroblasts exhibited a significant increase in the fraction of G1 phase cells $(P<$ $0.05)$ and a corresponding reduction in the proportion of G2-M phase cells $(P<0.05)$. These data suggest that ARID1A duplication in patient-derived fibroblasts may slow cell-cycle progression from $\mathrm{G} 1$ to $\mathrm{S}$ phase.

\section{DISCUSSION}

Somatic and germ-line mutations in SWI/SNF components have previously been implicated in tumor development. ${ }^{15}$ Recently, heterozygous germ-line mutations in components of the BAF complex were identified in patients with nonsyndromic intellectual disability, CSS, and Nicolaides-Baraitser syndrome (OMIM 601358). ${ }^{8}$ The common denominators of the phenotypes of these patients are severe intellectual disability and speech delay. To date, only truncating mutations leading to a loss of function of the ARIDIA gene have been reported. ${ }^{8}$ Here, we present four patients with 1p36.11 duplications detected by array-comparative genomic hybridization. These patients shared a similar phenotype, consisting primarily of microcephaly, intellectual disability, delayed motor milestones, hand and foot anomalies, growth impairment, constipation, frequent airway infections, dysmorphic facial features, and stereotypies. They all have a characteristic nasal tip with notched alae nasi and a low columella (Figure 1a). Deficiency of the lateral vermillion border of the upper lip is also seen (Figure 1a), as well
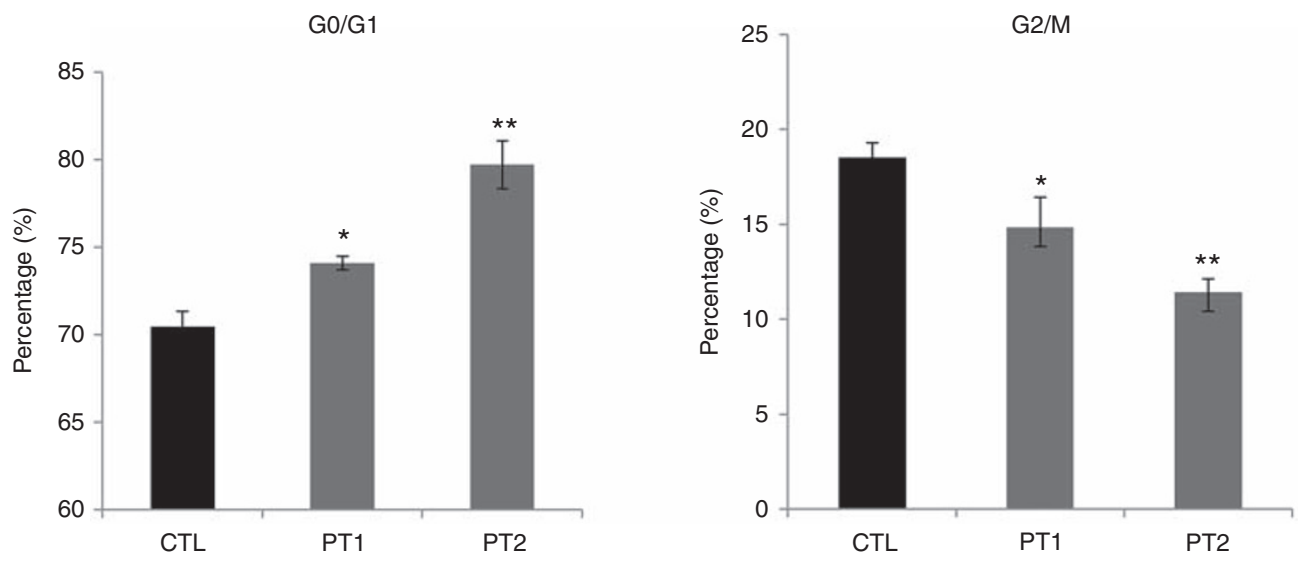

Figure 4 ARID1A overexpression induced G0/G1 cell-cycle arrest. Control (CTL), patient 1 (PT1), and patient 2 (PT2) cells were stained with propidium iodide and analyzed by flow cytometry. The percentage of cells in GO/G1 phase was significantly higher in the patient (gray) group than in the control group (black). A reciprocal reduction of cells in G2/M phase was observed in patient samples. Each experiment was performed in triplicate. ${ }^{*}$ Significant at $P<0.05$, $t$-test; **significant at $P<0.01, t$-test. 
as a high forehead and sparse hair (Figure 1a). The feet are very distinctive, with brachydactyly and shortening and medial deviation of the great toes (Figure 1a). We found a 122-kb MCR between these patients. Two genes were included in the MCR, but only the ARIDIA gene was fully duplicated; therefore, it seemed to be the best candidate to explain the clinical features. The PIGV gene is only partially included, and mutations in each $P I G V$ allele (homozygous or compound heterozygous) are necessary to substantially reduce the level of $P I G V$ protein expression or to be deleterious. ${ }^{16}$ In addition, PIGV expression did not appear to be deregulated in our patients according to RNA-seq and qRT-PCR experiments (Supplementary Table S4 online). Therefore, partial or complete duplication of PIGV is unlikely to be pathogenic. ${ }^{14}$

Conversely, ARID1A-a chromatin-remodeling gene with a dose-dependent effect because haploinsufficiency can cause CSS, ${ }^{5}$ - shows the highest likelihood of being a triplosensitive gene. Our previous and present studies revealed that ARIDIA duplication leads to a 1.5 -fold overexpression in patients (Supplementary Table S4 online). ${ }^{14}$ Subsequent western blot analysis was consistent with this modest gene overexpression detected by quantitative PCR and confirmed that ARID1A protein expression was slightly but significantly increased in fibroblasts from PT1 and PT2 (Figure 3b). Consequently, it is possible that duplication of the ARID1A gene could lead to a gain of function of the protein with enhanced activity. This hypothesis is supported by our present RNA-seq analyses that showed the strong deregulation of many genes involved in several biological functions (cell cycle, cell proliferation, and cell death as well DNA replication, recombination, and repair) for which ARID1A was previously demonstrated to be a key transcriptional regulator. ${ }^{17}$

Two larger duplications that fully include ARID1A are reported in the DECIPHER database. The first is an $18.9-\mathrm{Mb}$ duplication (ID 306661) that encompasses 314 genes; the second (ID 30771) is a 1.8-Mb duplication that encompasses 34 genes. Clinical information was available only for patient 30771, who presented with microcephaly, motor delay, fifth-finger clinodactyly, and $2 / 3$ toe syndactyly. Even if the genotype-phenotype correlation for such large duplications is uncertain, we can note that some clinical features presented by patient 30771 , such as microcephaly and hand/foot anomalies, are common to those found in our four patients.

Finally, we note that no duplications that fully included ARIDIA were reported in databases reporting copy-number variations from healthy subjects, such as the Database of Genomic Variants. In light of these considerations, there is strong evidence that ARID1A duplications are pathogenic and likely causative for the phenotype reported in the four patients.

An increasing number of observations in the literature demonstrate a close link between the constitutional alteration of tumor suppressor genes and neurodevelopmental disorders. ${ }^{18,19}$ Nevertheless, the molecular mechanisms linking the increase of ARID1A gene product with the reported clinical features remain to be understood. In our study, we observed that ARID1A duplication in patient-derived fibroblasts slowed cell-cycle progression from G1 to $S$ phase (Figure 4). This result was consistent with the expected role of ARID1A in the cellcycle checkpoint machiner ${ }^{20}$ and with the body of oncological literature showing that the overexpression of ARID1A leads to an arrest of cell-cycle progression in G1/S phase. ${ }^{21}$

Interestingly, it has been demonstrated that the BAF complex is involved in the proliferation and differentiation of neural stem and progenitor cells. ${ }^{8,22}$ Moreover, it is understood that the brain's size at birth is dependent on the ability of neuroprogenitor cells to proliferate and self-renew..$^{23,24}$ Therefore, a slight perturbation in the number of cell divisions of progenitor and stem cells, such as slowed cell-cycle progression, can have dramatic effects on brain size and may lead to microcephaly. ${ }^{25}$ Alterations in cell-cycle control and kinetics with a prolonged G1 phase reported in patient-derived fibroblasts may thus be a major factor in intellectual disability and microcephaly. However, cellcycle alterations shown in patient-derived fibroblasts could be different in neural progenitors and should be further evaluated.

From a molecular point of view, IPA analysis of our RNA-seq data showed significant enrichment of genes related to several developmental disorders reported in all our patients, such as congenital malformations of the skeleton $\left(P=4.13 \mathrm{e}^{-6}, z\right.$-score: 2.146), craniofacial abnormalities $\left(P=5.49 \mathrm{e}^{-6}, z\right.$-score: 2.176$)$, growth failure $\left(P=6.16 \mathrm{e}^{-5}, z\right.$-score: 4.002$)$, microcephaly $(P=$ $8.54 \mathrm{e}^{-5}, z$-score: 2.630 ), and congenital malformations of brain $\left(P=2.76 \mathrm{e}^{-4}, z\right.$-score: 2.534$)$. Most intriguingly, transcriptional analysis also showed that the majority of the known microcephaly-associated genes were upregulated in our patients' fibroblasts (Figure 2a, Supplementary Table S2 online). ${ }^{24}$ Almost all of these genes are closely linked to cell-cycle regulation and/ or centrosomal functions and are involved in severe primary microcephaly. 26,27

Primary microcephaly is defined by an occipitofrontal circumference (OFC) less than the third percentile present at birth and should be distinguished from secondary microcephaly, which develops postnatally. ${ }^{28}$ In our patients, OFC at birth was available only for PT1, for whom it was at the 10th percentile. However, biometric parameters at the lower limit of the normal range were reported for PT2 without more details. At the time of the last clinical evaluation, three patients presented with an OFC lower than the third percentile and one with an OFC lower than the 10th percentile. Although some OFCs at birth were missing, these data may suggest that the phenotype of these patients is more likely to be secondary microcephaly. The biological and clinical impacts of the overexpression of these microcephaly-associated genes, most of which are unlikely to be dosage-sensitive, remain uncertain. However, we cannot exclude the hypothesis that overexpression of one or more of these genes could have a significant negative effect on common cellular functions or pathways but may be less dramatically harmful for the cell than the downregulation or the total absence of the encoded protein. This might partly explain why our patients present with a secondary microcephaly and not a primary microcephaly, but it remains to be demonstrated. 
Beyond the overexpression of some microcephaly-associated genes, IPA revealed that the mitotic role of polo-like kinase pathway was among the most statistically significant pathways deregulated in patients with microduplications encompassing ARIDIA $(P=4.96)$ and the only pathway with an absolute $z$-score $>2$ (2.673) (Figure 2a). Almost all altered transcripts of the PLK pathway in patients with ARID1A duplication were upregulated (Supplementary Table S3 online). ARID1Atargeted siRNA experiments in patient fibroblasts confirmed the existence of a strong link between variations in ARID1A expression and the expression level of several PLK pathway genes (Figure 3c). Although we cannot totally exclude the possibility that some differences between cases and controls (e.g., in age) may induce confounding factors, these data converged and support that the genomic ARID1A duplication could be directly responsible for the dysregulation of the mitotic role of polo-like kinase pathway observed with RNA-seq in patient fibroblasts. In the mitotic role of polo-like kinase pathway, PLK4 presents the highest gene overexpression detected by RNA-seq (FC >100) (Supplementary Table S3 online). Our transcriptomic data were confirmed by qRT-PCR and western blot analysis showing that PLK4 is also markedly increased at the protein level in patient fibroblasts (Figure $3 \mathbf{b}$ ).

Interestingly, upregulation of Plk4 was previously shown to impede brain development and cause microcephaly in mice. ${ }^{29}$ Furthermore, individuals with loss-of-function mutations in PLK4 display profound microcephaly, confirming that gene PLK4 dosage variations (loss or gain) are pathogenic and that both lead to microcephaly. ${ }^{30,31}$ Using qRT-PCR, we found that another PLK gene, the PLK1 gene, was strongly overexpressed in our patients. Experiments with different animal models have demonstrated that gene dosage variations of PLK1 can also have a critical effect on cortical neurogenesis, the regulation of mammalian brain size, and the pathogenesis of congenital neurodevelopmental disorders, such as microcephaly. ${ }^{32,33}$ In addition, in 2013, Barber et al. ${ }^{34}$ described two patients with intellectual disability and dysmorphic features with a 16p11.2p12.2 duplication including PLK1, which seems to be one of the best candidate genes to explain the phenotype. These patients present with the most clinical features observed in our four patients with ARID1A duplications, such as developmental and psychomotor delay, microcephaly, growth restriction, stereotyped behaviors, dysmorphic features, ear anomalies, feet and hand anomalies, and recurrent infections. ${ }^{34}$

Interestingly, the reciprocal 16p11.2-p12.2 deletion leads to microcephaly, suggesting that both overexpression and decreased expression of some genes can lead to a reciprocal effect such as microcephaly or macrocephaly. This mirror effect was not observed in our case because duplications or inactivating mutations of the ARID1A gene lead to similar defects such as microcephaly. Moreover, many other clinical features, such as developmental delay, hand and foot anomalies, growth impairment, gastrointestinal problems, and spinal malformations, are common in CSS patients with the 1 p36.11 duplication. The same observations were made for other reciprocal deletions and duplications at several loci, such as $7 \mathrm{q} 11.23$, $22 \mathrm{q} 11.2,16 \mathrm{p} 13.3$, and 17p11.2. ${ }^{35}$ The defects observed in these reciprocal genomic disorders may identically hamper normal cell functions and involve the same organs. ${ }^{36}$ Additional experiments, such as comparative transcriptomic studies involving patients with reciprocal genomic disorders, would be required to explore in depth the functional link between gene dosage variations of some genes and the reported phenotype.

Finally, we note that some atypical clinical features were found in only a single patient among the four patients with 1p36.11 duplication (Table 1). This could suggest that other gene(s) included in the duplication may participate in the patients' phenotype. Moreover, we cannot rule out a positional effect of the duplication that may disturb other genes located in the vicinity of ARID1A and participate in the phenotype. ${ }^{37,38}$ Further investigations could be initiated to exclude the implication of other genes outside the MCR or a potential positional effect in the clinical features.

In conclusion, our study shows, for the first time, the clinical and molecular descriptions of a new microduplication syndrome involving the ARIDIA gene. Although additional work will be necessary to completely elucidate the effects of abnormal ARIDIA copy-number variations, we provide here the first step to clarifying the pathophysiological mechanism connecting ARID1A gene dosage with intellectual disability and microcephaly.

\section{SUPPLEMENTARY MATERIAL}

Supplementary material is linked to the online version of the paper at http://www.nature.com/gim

\section{ACKNOWLEDGMENTS}

The authors are grateful to all the patients, their families, and the clinicians involved for their cooperation.

\section{DISCLOSURE}

The authors declare no conflict of interest.

\section{REFERENCES}

1. Euskirchen G, Auerbach RK, Snyder M. SWI/SNF chromatin-remodeling factors: multiscale analyses and diverse functions. J Biol Chem 2012;287:30897-30905.

2. Brownlee PM, Meisenberg C, Downs JA. The SWI/SNF chromatin remodelling complex: Its role in maintaining genome stability and preventing tumourigenesis. DNA Repair (Amst) 2015;32:127-133.

3. Jones S, Li M, Parsons DW, Zhang X, Wesseling J, Kristel P, et al. Coffin-Siris syndrome and related disorders involving components of the BAF (mSWI/ SNF) complex: historical review and recent advances using next generation sequencing. Am J Med Genet C Semin Med Genet 2014;166C:241-51.

4. Biegel JA, Busse TM, Weissman BE. SWI/SNF chromatin remodeling complexes and cancer. Am J Med Genet C Semin Med Genet 2014;166C:350-366.

5. Tsurusaki $\mathrm{Y}, \mathrm{Okamoto} \mathrm{N}$, Ohashi $\mathrm{H}$, et al. Mutations affecting components of the SWI/SNF complex cause Coffin-Siris syndrome. Nat Genet 2012;44:376-378.

6. Coffin GS, Siris E. Mental retardation with absent fifth fingernail and terminal phalanx. Am J Dis Child 1970;119:433-439.

7. Kosho T, Miyake N, Carey JC. Coffin-Siris syndrome and related disorders involving components of the BAF (mSWI/SNF) complex: historical review and recent advances using next generation sequencing. Am J Med Genet C Semin Med Genet 2014;166C:241-251. 


\section{ORIGINAL RESEARCH ARTICLE}

8. Santen GW, Aten E, Sun Y, et al. Mutations in SWI/SNF chromatin remodeling complex gene ARID1B cause Coffin-Siris syndrome. Nat Genet 2012;44:379380.

9. Santen GW, Aten E, Vulto-van Silfhout AT, et al.; Coffin-Siris consortium. CoffinSiris syndrome and the BAF complex: genotype-phenotype study in 63 patients. Hum Mutat 2013;34:1519-1528.

10. Tsurusaki Y, Okamoto N, Ohashi H, Mizuno S, Matsumoto N, Makita Y, et al. Coffin-Siris syndrome is a SWI/SNF complex disorder. Clin Genet 2013;85:1-7.

11. Wieczorek D, Bögershausen N, Beleggia F, et al. A comprehensive molecular study on Coffin-Siris and Nicolaides-Baraitser syndromes identifies a broad molecular and clinical spectrum converging on altered chromatin remodeling. Hum Mol Genet 2013:22:5121-5135.

12. Mari F, Marozza A, Mencarelli MA, et al. Coffin-Siris and Nicolaides-Baraitser syndromes are a common well recognizable cause of intellectual disability. Brain Dev 2015;37:527-536.

13. Son EY, Crabtree GR. The role of BAF (mSWI/SNF) complexes in mammalian neural development. Am J Med Genet C Semin Med Genet 2014;166C: 333-349.

14. Coutton C, Bidart M, Rendu J, et al. 190-kb duplication in 1 p36.11 including PIGV and ARID1A genes in a girl with intellectual disability and hexadactyly. Clin Genet 2013;84:596-599.

15. Romero OA, Sanchez-Cespedes M. The SWI/SNF genetic blockade: effects in cell differentiation, cancer and developmental diseases. Oncogene 2014;33: 2681-2689.

16. Krawitz PM, Schweiger MR, Rödelsperger $C$, et al. Identity-by-descent filtering of exome sequence data identifies PIGV mutations in hyperphosphatasia mental retardation syndrome. Nat Genet 2010;42:827-829.

17. Wu RC, Wang TL, Shih leM. The emerging roles of ARID1A in tumor suppression. Cancer Biol Ther 2014;15:655-664.

18. Bidart M, Coutton C. Intellectual disability, oncogenes and tumour suppressor genes: the way forward? J Genet 2012;91:257-258.

19. Santen GW, Kriek M, van Attikum H. SWI/SNF complex in disorder: SWltching from malignancies to intellectual disability. Epigenetics 2012;7:1219-1224.

20. Shigetomi $H$, Oonogi $A$, Tsunemi $T$, et al. The role of components of the chromatin modification machinery in carcinogenesis of clear cell carcinoma of the ovary (review). Oncol Lett 2011;2:591-597.

21. Zeng Y, Liu Z, Yang J, Liu Y, Huo L, Li Z, et al. ARID1A is a tumor suppressor and inhibits glioma cell proliferation via the PI3K pathway. Head Neck Oncol 2013;5:6.

22. Lessard J, Wu J, Ranish JA, et al. An essential switch in subunit composition of a chromatin remodeling complex during neural development. Neuron 2007;55:201-215.
23. Rakic P. A small step for the cell, a giant leap for mankind: a hypothesis of neocortical expansion during evolution. Trends Neurosci 1995;18:383-388.

24. Barbelanne M, Tsang WY. Molecular and cellular basis of autosomal recessive primary microcephaly. Biomed Res Int 2014;2014:547986.

25. Bicknell LS, Walker S, Klingseisen A, et al. Mutations in ORC1, encoding the largest subunit of the origin recognition complex, cause microcephalic primordial dwarfism resembling Meier-Gorlin syndrome. Nat Genet 2011:43:350-355.

26. Ostergaard P, Simpson MA, Mendola A, et al. Mutations in KIF11 cause autosomal-dominant microcephaly variably associated with congenital lymphedema and chorioretinopathy. Am J Hum Genet 2012;90:356-362.

27. Morris-Rosendahl DJ, Kaindl AM. What next-generation sequencing (NGS) technology has enabled us to learn about primary autosomal recessive microcephaly (MCPH). Mol Cell Probes 2015:29:271-281.

28. Woods CG. Human microcephaly. Curr Opin Neurobio/ 2004;14:112-117.

29. Marthiens V, Rujano MA, Pennetier C, Tessier S, Paul-Gilloteaux P, Basto R. Centrosome amplification causes microcephaly. Nat Cell Biol 2013;15: 731-740.

30. Martin CA, Ahmad I, Klingseisen A, et al. Mutations in PLK4, encoding a master regulator of centriole biogenesis, cause microcephaly, growth failure and retinopathy. Nat Genet 2014;46:1283-1292.

31. Shaheen R, Al Tala S, Almoisheer A, Alkuraya FS. Mutation in PLK4, encoding a master regulator of centriole formation, defines a novel locus for primordial dwarfism. J Med Genet 2014:51:814-816.

32. Kishi $K$, van Vugt MA, Okamoto $K$, Hayashi $Y$, Yaffe MB. Functional dynamics of Polo-like kinase 1 at the centrosome. Mol Cell Biol 2009:29:3134-3150.

33. Sakai D, Dixon J, Dixon MJ, Trainor PA. Mammalian neurogenesis requires Treacle-Plk1 for precise control of spindle orientation, mitotic progression, and maintenance of neural progenitor cells. PLoS Genet 2012;8:e1002566.

34. Barber JC, Hall V, Maloney VK, et al. 16p11.2-p12.2 duplication syndrome; a genomic condition differentiated from euchromatic variation of 16p11.2. Eur J Hum Genet 2013:21:182-189.

35. Tassano E, Alpigiani MG, Calcagno A, et al. Clinical and molecular delineation of a 16p13.2p13.13 microduplication. Eur J Med Genet 2015;58:194-198.

36. Pérez de Castro I, de Cárcer G, Malumbres M. A census of mitotic cancer genes: new insights into tumor cell biology and cancer therapy. Carcinogenesis 2007:28:899-912

37. Kleinjan DJ, van Heyningen V. Position effect in human genetic disease. Hum Mol Genet 1998:7:1611-1618.

38. Blumenthal I, Ragavendran A, Erdin S, et al. Transcriptional consequences of $16 p 11.2$ deletion and duplication in mouse cortex and multiplex autism families. Am J Hum Genet 2014;94:870-883. 